Published in final edited form as:

AEA Pap Proc. 2019 May ; 109: 344-349. doi:10.1257/pandp.20191079.

\title{
A Well-Being Snapshot in a Changing World
}

\author{
Daniel J. Benjamin, \\ Center for Economic and Social Research, University of Southern California, 635 Downey Way, \\ Suite 312, Dauterive Hall, Los Angeles, CA 90089, and NBER \\ Kristen B. Cooper, \\ Department of Economics and Business, Gordon College, 255 Grapevine Road, Wenham, MA \\ 01984
}

\section{Ori Heffetz,}

Department of Economics and Federmann Center for the Study of Rationality, The Hebrew University of Jerusalem, S.C. Johnson Graduate School of Management, Cornell University, 324 Sage Hall, Ithaca, NY 14853, and NBER

\section{Miles Kimball ${ }^{\star}$ \\ Department of Economics, University of Colorado Boulder, 256 UCB, Boulder, CO, 80309, and NBER}

Over the last few decades, globalized, technology-driven growth has enabled U.S. consumers to experience new, improved, and cheaper goods and services. These range from better medical procedures, healthier foods, and safer transportation, to ubiquitous digital communication and on-demand entertainment. The emergence of the "gig economy" (i.e., on-demand, online labor markets) has given workers increased autonomy, flexibility, and independence. More broadly, the societal and cultural change that accompanies globalization, technological progress, and economic growth, is often characterized by increased liberties, openness, social development, and empowerment.

However, the same forces of globalization and technological progress that have brought these improvements can also have negative impacts on well-being. Price-reducing geographical flexibility in production may increase worker anxiety. Efficiency-increasing artificial-intelligence systems may threaten workers' jobs. The gig economy may result in workers giving up many of the benefits of a full-time job. The societal changes that empower some may leave others feeling disoriented, disconnected, and powerless.

The fruits and costs of these economic and social transformations may be borne disproportionately by different groups. When studying the distributional consequences of growth, economists traditionally focus on market goods—or the income that enables their consumption —in part because these data are most readily available. Following Easterlin (1974), a growing number of economists have also studied self-reported well-being, typically focusing on responses to a single survey question, or a small number of questions —again, in large part because of data availability.

*Kimball: (miles.kimball@colorado.edu). 
In this paper, we analyze data from a survey in which we asked respondents to self-report how they rate on one or more of 204 aspects of well-being. The 204 aspects are those that we expected to be most closely related to globalization and technology-driven growth among a much larger set of over 2,000 aspects of well-being included in our survey. The larger set is intended to be a reasonably comprehensive list of "fundamental" goods-goods that are the ultimate objects of desire (c.f., Lancaster, 1966). Consumption of market goods, time, and other intermediate aspects of well-being are viewed as inputs into these final goods (for a formal framework, see Benjamin et al., 2014).

We ask how different groups in the U.S. population differ in their self-reported levels of these 204 aspects.

\section{Survey Overview}

We ran our survey on Amazon's Mechanical Turk (MTurk) platform in five waves between December, 2016 and August, 2017. Our sample is demographically diverse but not nationally representative (see online appendix table A1).

While our survey includes measures of additional aspects of well-being and other types of questions, in this paper we focus on self-reported ratings of the 204 aspects discussed below. Each rating question asks: "Thinking about the past year, how would you rate [Aspect of well-being]?" Respondents move a horizontal slider, where response options are integers from 0 to 100. We experimentally varied the labels on some response options, and we pool the data across these variations; see online appendix for screenshots and further details, and see Benjamin et al. (2017) for a discussion of how we developed the full list of 2,000+ aspects of well-being.

We analyze data from 1,576 respondents who self-rated at least one of the 204 aspects. ${ }^{1}$ Respondents were paid by the number of questions they answered, and chose when to stop. The median respondent rated 6 of the 204 aspects ( mean $=23, \mathrm{SD}=42$ ).

We aggregated the 204 aspects into seven themes (see table 1 for a complete list):

- $\quad$ Satisfaction: 33 broad, evaluative aspects, e.g., "how satisfied you are with your life," "your overall well-being," and "you achieving the things in your life that are worth wanting to achieve."

- Affect: 31 positive and absence-of-negative aspects, e.g., "how happy you feel," "how often you feel good," and "you not feeling anxious."

- Growth: 21 items related to perceptions about economic, technological, and knowledge-based growth, e.g., "how quickly science is advancing," "the total size of your nation's economy (GDP)," and "how fast technology and productivity are improving."

\footnotetext{
${ }^{1}$ In survey waves $2-5$, aspects were sampled at random from our full 2,000+ list. In wave 1, a fixed set of 27 aspects was rated by all respondents. Five of the 27 are among the 204 aspects we study here and are therefore oversampled relative to the other 199 aspects.
} 
- Autonomy: 31 aspects related to the autonomy, control, and flexibility offered by the gig economy, e.g., "you being your own boss," "you having autonomy in your job," and "you having control over your own time at work."

- Job: 32 aspects of workplace well-being that apply also, or even mainly, to the traditional economy, e.g., "the overall quality of your experience at work," "you and your coworkers working together as an effective team," and "you feeling that your work has value."

- Calmness: 28 aspects, e.g., "the amount of stability in your life," "you not feeling under constant strain," and "you not feeling restless."

- Belonging: 28 aspects, e.g., "your sense of community," "you fitting in," and "you feeling you belong."

We constructed each theme's list based on the concepts captured by the aspects' wording; we did not check how varying the lists would Affect the findings below.

The first two themes, Satisfaction and Affect, correspond to the two types of self-reported well-being measures that are most widely used: "evaluative" measures of overall well-being (e.g., a question about life satisfaction) and "Affective" measures of general emotional states (e.g., happiness and anxiety). These two themes allow us to compare the demographic differences in our sample with those reported in the literature, including in more nationally representative samples.

The third theme, Growth, includes perceptions of things that can be objectively quantified, such as economic growth, scientific advance, and technological progress. We included them as aspects of well-being because they may be public goods that are valued in themselves. To the extent that perceptions about their objective quantities are widely shared, we do not expect substantial demographic differences in their ratings.

The last four themes capture aspects of well-being that are especially likely to be impacted by economic growth, technological change, and labor-market disruptions: Autonomy and Job correspond to aspects directly related to workers' well-being, while Calmness and Belonging capture specific positive feelings that may be disrupted by rapid change.

For respondent distributions of mean aspect ratings by theme across demographic groups, see online appendix table A2.

\section{Analysis and Findings}

We analyze our data with seven regressions. Each dependent variable is one theme's aspect ratings, demeaned at the aspect level. The independent variables are: continuous variables for age, age ${ }^{2}$, and log income, and indicator variables for all other demographic categories (see below). Standard errors are clustered by respondent.

Table 2 presents our results. To deal with multiple hypothesis testing, we assess "statistical significance," which we indicate with an asterisk, using a false discovery rate (FDR) threshold of 10\%, calculated using the Benjamini and Hochberg (1995) algorithm. Rather 
than controlling the Type I error rate for any particular coefficient, the FDR threshold ensures that the null hypothesis is true (in expectation) for at most $10 \%$ of the coefficients we indicate as significant.

Using the same FDR threshold with two new sets of hypotheses, we also indicate, with a dagger, coefficients that are statistically different from their corresponding Satisfaction coefficient and, with a double dagger, Job coefficients that are statistically different from their corresponding Autonomy coefficients; see online appendix section A3 for stackedregression specifications. As a broad, widely used measure, Satisfaction is a natural benchmark for comparisons; we compare Autonomy and Job because growth and technology might increase autonomy and flexibility while worsening other aspects of workplace well-being.

\section{A. Evaluative and Affective Self-Reported Well-Being}

Table 2's first two columns broadly replicate standard findings on demographic differences in evaluative and Affective well-being measures (see, e.g., Clark, 2018). In both columns, we find that measured well-being has a U-shaped relationship with age; is increasing with $\log$ income (but statistically less so for Affect, which is not statistically different from zero using the FDR threshold of 10\%); is lower among those who have no romantic partner and those who are not employed full time (although, again, some of the negative coefficients do not reach the FDR threshold); and is higher among those for whom religion is at least a little important. This qualitative replication of findings from standard single-question measures in representative samples is reassuring.

\section{B. Growth-Related Perceptions}

As shown in the third column, the coefficients in the Growth regression are generally small, are never statistically different from zero, and are often statistically significantly smaller than those in the Satisfaction column. Thus, we find no detectable demographic differences in perceptions of growth-related quantities, suggesting these perceptions are broadly shared. We interpret this result as passing a reasonable falsification test: the lack of demographic associations for Growth provides reassurance that artifactual differences across demographic groups (e.g., different ways of using the 0-100 response scale) are not driving the differences that we observe for other themes.

\section{Autonomy-, Job-, Calmness-, and Belonging-Related Aspects of Well-Being}

Our main results are reported in the fourth through seventh columns. We highlight three findings.

First, all coefficients corresponding to the demographics that are significant for Satisfaction - age $^{2}$, income, no romantic partner, and religion - have the same sign as Satisfaction but are almost always smaller, often statistically significantly so, and are often not significantly different from zero. This pattern indicates that these demographics' relations with Autonomy, Job, Calmness, and Belonging are weaker than with Satisfaction. But the demographic relationships for Satisfaction, which are often similar for Affect (though with a flatter income gradient), are among those that have received the most attention in the 
subjective well-being literature. We find that when we examine our more specific dimensions of self-reported well-being, some of these relationships are less dominant.

Second, being unmarried (with or without a partner) is substantially less negatively associated with Autonomy, Job, Calmness, and Belonging than it is with Satisfaction, almost always statistically so. The association may even flip sign for the partnered unmarried, though not statistically significantly. We discuss this finding below.

Third, while all themes but Growth are negatively associated with not being employed fulltime, the most (and almost only) statistically significant associations are for Job. Almost all other coefficients are substantially smaller (in absolute value). In particular, consider those neither full-time employed nor unemployed, in the "Other empl.status" row. They include part-time employees, disabled, homemakers, and students-those who may benefit most from the emergence of the gig economy. Indeed, the large negative coefficient on Job contrasts with a coefficient on Autonomy that is not statistically different from zero, and is statistically smaller than the coefficients on both Job and Satisfaction.

\section{Discussion}

We would like to answer the question of how recent economic growth has causally affected various aspects of well-being. We cannot answer it because we have cross-sectional data, rather than a panel, with no source of exogenous variation in growth. Nonetheless, the analysis we conduct-examining demographic differences in aspects of well-being in a convenience sample at a single point in time-might be useful for singling out aspects, or aspect themes, that should begin to be measured in panel data. Indeed, our findings of somewhat different demographic associations for Autonomy, Job, Calmness, and Belonging relative to the standard self-reported well-being measures suggest that these aspects might also be Affected differently by experimental or policy interventions.

Our findings that different aspects (or themes) are distributed somewhat differently in the population raises a natural question: Which dimensions of well-being are important to people? In Benjamin et al. (2014), we make some initial progress on this question by asking people to make tradeoffs between different aspects of well-being, and using them to estimate the aspects' marginal rates of substitution. We asked such trade-off questions also in the present survey. As we collect more data, we hope to be able to address the question of how people value the aspects relative to each other.

Another natural question is why different groups have different levels of the aspects. Viewed through the lens of a model of home production, this question becomes: Do groups with lower levels of a particular aspect of well-being produce less of it due to preferences or due to constraints? The answer may have policy implications. Consider people with no romantic partner, who rate themselves much lower than others on Satisfaction but significantly less so on Autonomy and Job. If their relatively better ratings on work-related well-being reflect that they care a lot about it (and therefore invest in it), then they might be hit harder than others by job loss or insecurity. In contrast, if their ratings reflect a relative advantage in producing work-related well-being rather than general evaluative well-being, then a decrease 
in job quality may decrease their well-being by less. Investigating whether different levels of aspects are driven by such "demand" or "supply" factors is left for future research.

\section{Supplementary Material}

Refer to Web version on PubMed Central for supplementary material.

\section{Acknowledgments}

We are grateful for NIH/NIA grant R01-AG051903 to the University of Southern California for financial support. We thank Jana Gallus for helpful comments.

\section{REFERENCES}

Benjamin Daniel J., Cooper Kristen B., Heffetz Ori, and Kimball Miles S.. 2017 "Challenges in Constructing a Survey-Based Well-Being Index.” American Economic Review Papers and Proceedings, 107(5): 81-85.

Benjamin Daniel J., Heffetz Ori, Kimball Miles S., and Szembrot Nichole. 2014 "Beyond Happiness and Satisfaction: Toward Well-Being Indices Based on Stated Preference." American Economic Review 104(9): 2698-2735. [PubMed: 25404760]

Benjamini Yoav, and Hochberg Yosef. 1995 "Controlling the False Discovery Rate: A Practical and Powerful Approach to Multiple Testing." Journal of the Royal Statistical Society Series B 57(1): 289-300.

Clark Andrew E. 2018 “Four Decades of the Economics of Happiness: Where Next?" Review of Income and Wealth 64(2): 245-269.

Easterlin Richard. 1974 "Does Economic Growth Improve the Human Lot? Some Empirical Evidence" In David Paul A. and Reder Melvin W.. Nations and Households in Economic Growth: Essays in Honor of Moses Abramovitz. New York: Academic Press, Inc.

Lancaster Kelvin J. 1966 “A New Approach to Consumer Theory.” Journal of Political Economy 74(2): 132-157. 


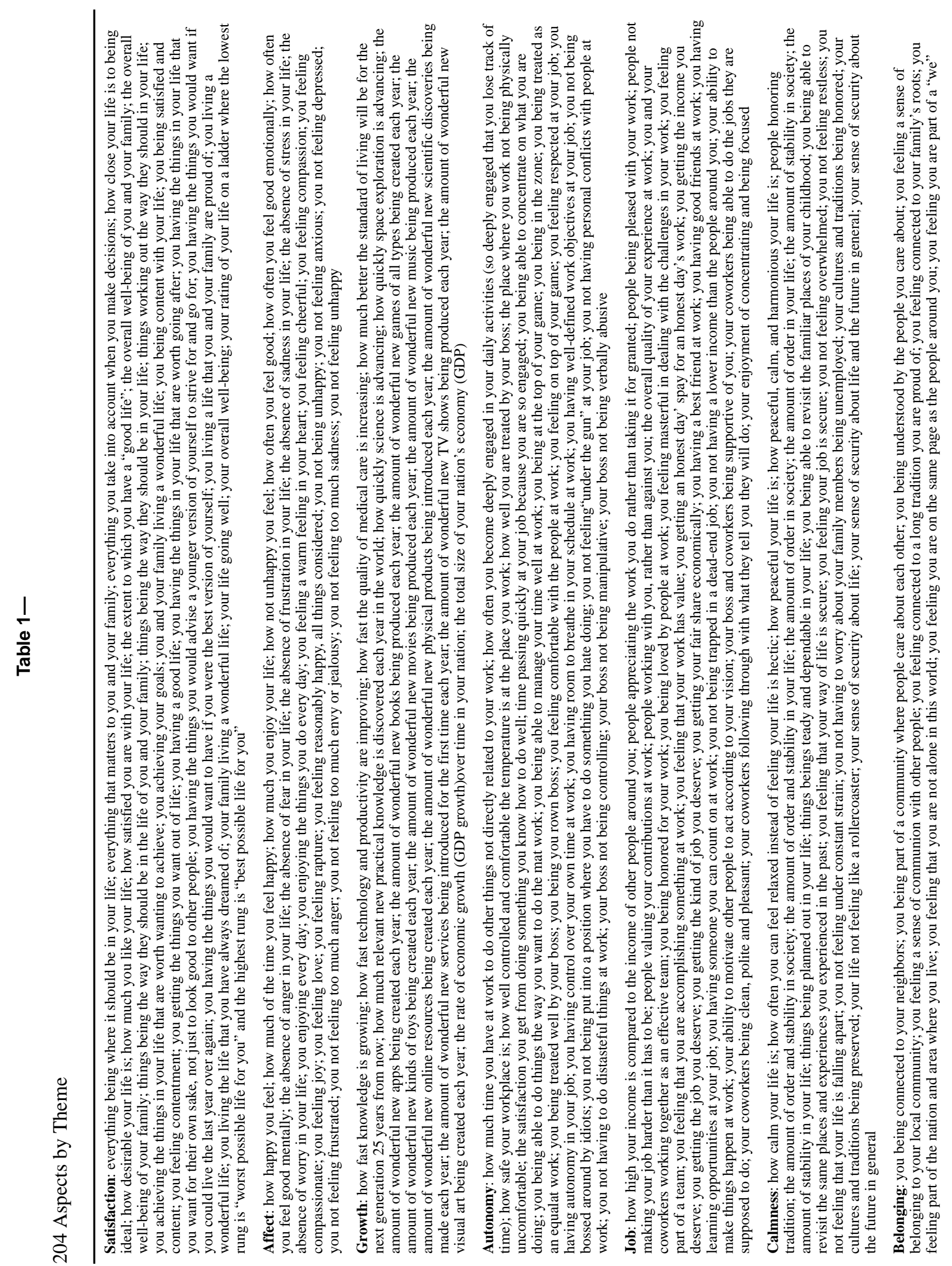




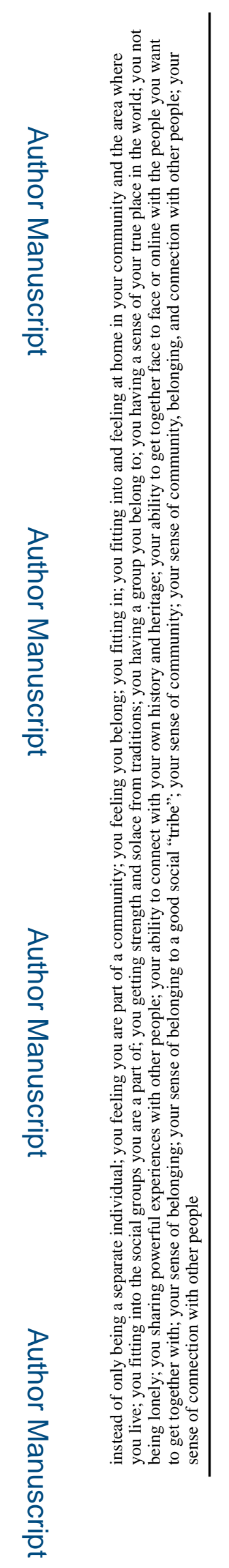

AEA Pap Proc. Author manuscript; available in PMC 2020 March 20. 


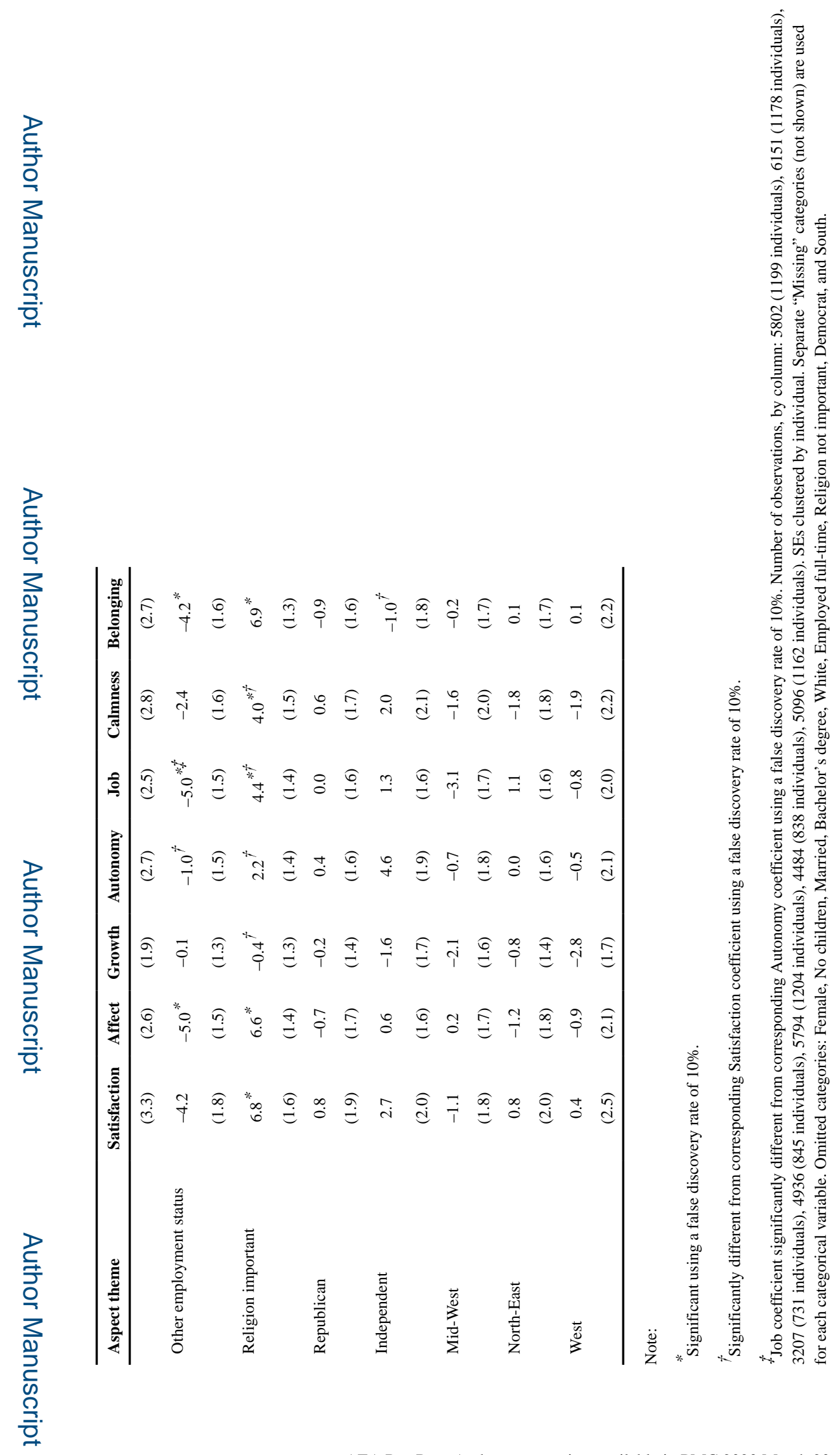

